\title{
Estrategias en la introducción y uso del e.Learning en educación superior
}

Josep M Duart

Universitat Oberta de Catalunya (UOC)

\section{ALGUNAS IDEAS SOBRE EL CONCEPTO DE E.LEARNING}

Son muchas las definiciones y traducciones más o menos literales del término e.Learning. Desde aprendizaje electrónico a virtual pasando por aprendizaje flexible. No pretendemos, aquí y ahora, ni definir ni proponer un nuevo término, pero sí que debemos precisar el marco semántico del mismo, al menos para comprender y compartir el ámbito conceptual sobre el que estamos hablando. Una aproximación conceptual, de elaboración propia, podría ser la siguiente: una metodología de enseñanza-aprendizaje basada en el uso de contenidos educativos distribuidos a través de

Correspondencia:

J. M. Duart

Director de la Cátedra UNESCO de e.Learning de la UOC

Avda. Tibidabo 39

08035 - Barcelona

jduart@uoc.edu un soporte electrónico, fundamentalmente Internet. Dicho en palabras más sencillas, "acceso online a recursos de aprendizaje, desde cualquier sitio y a cualquier hora". Se trata, en definitiva de relacionar, a mi entender, tres ideas clave: una metodología de enseñanza y de aprendizaje; unos contenidos educativos; y el potencial del acceso a la información via Internet. La relación entre estas tres ideas, a parte de sugerente, es altamente compleja y da lugar a un sinfín de situaciones educativas posibles. Nuestro interés en este articulo es aproximarnos a esta complejidad, siempre desde el punto de vista institucional.

Antes quisiéramos desmitificar algunas ideas que existen en torno al mundo de las tecnologías relacionadas con el aprendizaje. Hoy, después de décadas de uso de Internet en educación ya nos hemos dado cuenta, por ejemplo, que esta modalidad no es válida únicamente para la educación a distancia, es valida para la formación a largo de la vida, la formación continua y un excelente y necesario complemento a la formación presencial de los jóvenes universitarios o profesionales por ejemplo. No se trata de una modalidad para llegar a los que están lejos, en la distancia; es una modalidad para aquellos que necesitan organizarse su bien más preciado, el tiempo, y por tanto los trabajadores, profesionales en activo, que mejoran su formación 
constantemente. Estudiar usando tecnologías no quiere decir que el aprendizaje es rápido o fácil. Cualquier proceso de aprendizaje, el virtual también, requiere esfuerzo y tiempo. Los espejismos de la tecnología pueden ser muy traicioneros para la imagen de la formación con uso intensivo de Internet, por ejemplo. Y otro mito es el del coste. Pareciera que la formación vía telemática debería ser más económica. Ya sabemos que no es así. No nos extenderemos en la desmitificación de estos elementos que ya son obvios. Preferimos destacar desde el inicio que la formación vía telemática de calidad es la que es resultado de una adecuada y reflexiva planificación estratégica institucional.

El simple acceso a la información o a contenidos educativos no presupone aprendizaje. Necesitamos una metodología específica que tenga en cuenta el contexto en el que se produce el proceso educativo, un contexto asíncrono, propiciado por Internet y sus sistemas de comunicación, o bien un contexto hibrido, resultado de la mezcla inteligente de la presencialidad y la no presencialidad. En el continuo ${ }^{2}$ que va des de la presencialidad hasta la no presencialidad en los procesos de formación los matices son muy diversos. En la figura 1 mostramos algunas formas de concretar esos matices.

Si tenemos en cuenta la organización lectiva de la formación, es decir, el tiempo que estudiante y profesor deben dedicar al desarrollo del proceso de enseñanza y aprendizaje en un curso concreto y que concretamos en unidades temporales llamadas créditos, pensamos que a lo largo del continuo dibujado podemos definir cinco espacios de uso:

a) Uso casi inexistente de Internet. Internet está presente, ya sea como oferta institucional a través de una cuenta de correo que la institución provee a estudiantes y profesores, o través del portal de la universidad y sus servicios. El uso de Internet se sitúa en el nivel personal y no incide en el proceso de enseñanza y aprendizaje. b) Uso de Internet como apoyo al proceso de enseñanza-aprendizaje. Se trata de la substitución de una tecnología por otra, es decir, de la fotocopiadora por los repositorios de materiales en Internet. Aquí podemos encontrar los bancos de recursos educativos, los links a páginas web o buscadores específicos, bibliotecas, etc.

c) Uso de Internet como base del proceso de enseñanza-aprendizaje. Se trata de acompañar la clase con el uso de la tecnología. Aquí podemos encontrar experiencia como el uso de forum online, discusiones online, trabajo cooperativo síncrono o asíncrono online, etc, pero sin abandono del horario de clase presencial preestablecido.

d) Uso de Internet como parte del proceso de enseñanza-aprendizaje. El horario lectivo del curso se divide en una parte presencial y otra online. Ambas partes forman parte total del curso y son evaluables. En la parte online se usan los recursos adecuados para su desarrollo. Presencialidad y virtualidad se complementan en un espacio híbrido.

e) Uso intensivo de Internet en el proceso de enseñanza-aprendizaje. Encontramos aquí los espacios de interacción correspondientes a los cursos totalmente online, en los que la presencia es inexistente.

Desde el simple uso del correo electrónico como apoyo a cuestiones originadas en el aula presencial hasta el uso intensivo de Internet en la formación superior existe un gran abanico de posibilidades metodológicas y educativas. Lo importante, a nuestro entender, es que esas posibilidades no son únicamente el resultado del emprenedurismo educativo de un profesor o de un colectivo de estudiantes; deben ser consecuencia de una estrategia institucional pensada y valorada a partir de la tipología de estudiantes a los que se atiende y del contexto en el que se trabaja, incluyendo este la tipología del contenido que se imparte. En otras palabras, el uso de

Figura 1. Modelos institucionales según uso docente de la red

FORMACIÓN PRESENCIAL
FORMACIÓN HIBRIDA
FORMACIÓN VIRTUAL

\begin{tabular}{|c|c|c|c|c|}
\hline Casi no se usa & Se usa como apoyo & Se usa como base & $\begin{array}{c}\text { Se como parte } \\
\text { del curso }\end{array}$ & $\begin{array}{c}\text { Se usa } \\
\text { intensivamente }\end{array}$ \\
\hline
\end{tabular}


Internet en formación superior debe ser contemplado desde la estrategia institucional y debe comprender políticas y planificaciones que contemplen los diferentes aspectos clave en cualquier proceso educativo: el que aprende, el que facilita el aprendizaje, los contenidos educativos y el contexto en el que se desarrolla.

\section{MODELOS INSTITUCIONALES EN EL USO DEL Q.LEARNING: EN BUSCA DEL EQUILIBRIO COMO RESULTADO}

El uso institucional del e.Learning debe ser el resultado de una estrategia institucional. Para ello es necesario tener en cuenta diferentes variables y como siempre en estos casos no existe un resultado único para todas las instituciones, si no que cada institución debe ser capaz de interpretar su realidad y sus necesidades y dotarse del modelo que mejor da respuesta a ellas. De ahí la necesidad de encontrar una fórmula basada en el equilibrio, en la capacidad armónica de respuesta a las diferentes fuerzas que se generan como resultado de la introducción de cualquier proceso de innovación.

Para ayudar a la definición de la estrategia institucional en la introducción y el uso del e.Learning proponemos tener en cuenta, a parte del contexto y

Figura 2 Ámbitos de la institución educativa en la Sociedad Red

La institución educativa en la sociedad red

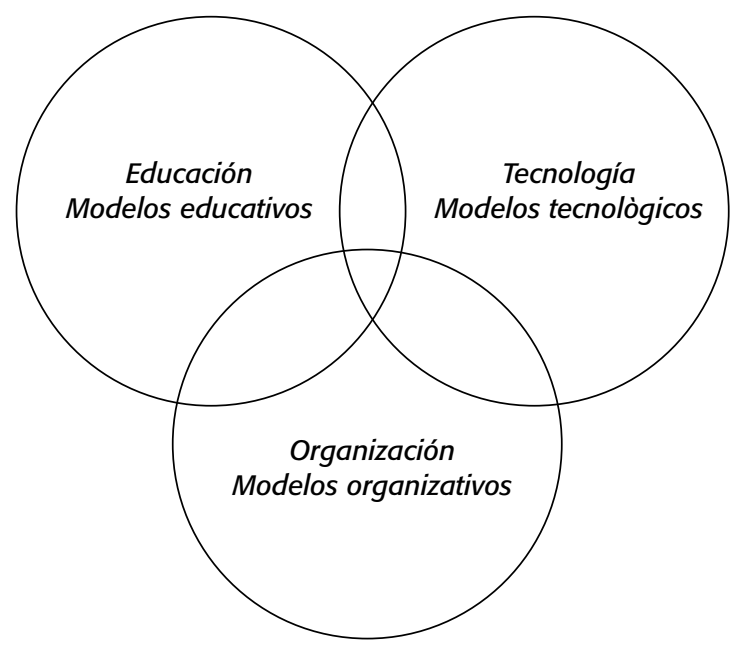

de la disciplina de aprendizaje, los siguientes elementos: la pedagogía o metodología de aprendizaje, la tecnología y la organización y administración del proceso diseñado. Estos tres elementos, como podemos observar en la figura 2, se interrelacionan entre si generando un sistema dinámico basado en la interacción y en la búsqueda del equilibrio. De ahí la necesidad de un tipo de dinámica institucional de geometría variable, es decir, flexible y con capacidad de adaptabilidad constante a una sociedad y a unos ciudadanos en constante proceso de cambio.

La institución debe ser capaz de definir su modelo educativo, su modelo tecnológico y su modelo organizativo, y todos ellos coordinados de forma coherente y equilibrada. ¿De qué depende esa definición de modelos y la consecución de ese equilibrio? A nuestro entender, de la capacidad de la institución para entender el perfil del usuario al que se dirige y valorar acertadamente sus necesidades formativas. No podemos pensar en modelos tecnológicamente avanzados dirigidos a profesionales con pocas capacidades o posibilidades de acceder a ellos o de sacarles el máximo provecho. $\mathrm{O}$ pensar en modelos educativos basados en la memorización cuando lo que se pretende, como habitualmente se trata en los profesionales de la salud, en el aprendizaje a partir de la resolución de problemas. De ahí que cada institución, de forma reflexiva, debe ser capaz de articular su propio modelo. $\mathrm{Y}$ el resultado de los modelos educativo, tecnológico y organitzativo debe ser, lógicamente, equilibrado y coherente. No tendría sentido un modelo basado en la asincronía total que no contemplase un sistema organizativo capaz de dar respuesta a las necesidades de los usuarios las 24 horas del día y todos los días del año. Y esta incoherencia, por ejemplo, es fácil de encontrar en sistemas educativos totalmente virtuales, especialmente en instituciones presenciales.

Si tenemos en cuenta la existencia de diferentes modelos para cada uno de los ámbitos mencionados y que además estos deben relacionarse entre si en búsqueda de un sistema equilibrado de interacción, comprenderemos que debemos tender hacía un modelo institucional que opere con coherencia en un entorno en el que presencia y no presencia actúan a la par buscando una tensión equilibrada, es decir, procurando un modelo híbrido.

Los cuadrantes dibujados a partir de los dos ejes reflejan el uso de la tecnología en la organización y en la formación estableciendo cuatro grandes tipo- 
Figura 3. Tipologías de institución educativa según el uso de la red.

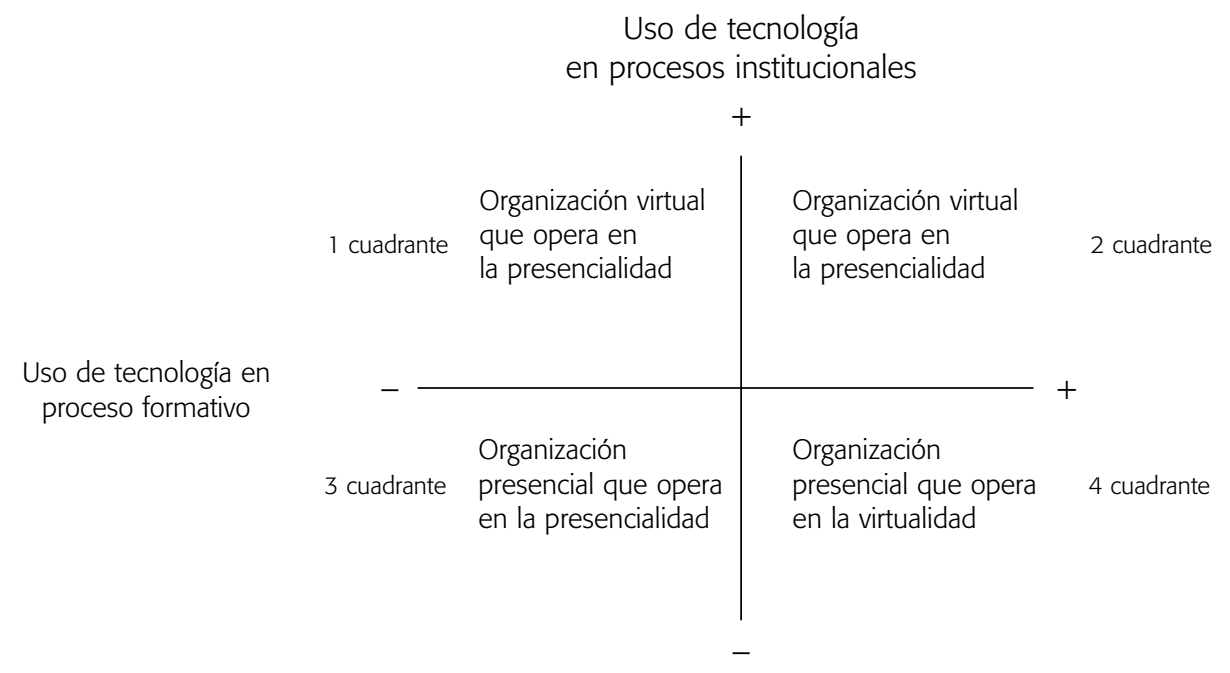

logías institucionales. Estos cuadrantes no nos marcan compartimentos estancos, sino que nos muestran unas líneas continuas que puede ir desde un uso intensivo de la tecnología hasta un uso puntual para determinados procesos.

Las organizaciones y los procesos educativos cuando se median por la tecnología sufren cambios y alteran sus mecanismos habituales de desarrollo, así como sus potenciales y posibilidades. No se organiza de igual forma la docencia virtual que la presencial, o la administración académica presencial que mediada por la tecnología. Tampoco será el mismo el público potencial al que nos dirigimos con cursos impartidos a través de Internet que con cursos presenciales o mixtos. La riqueza de matices y de posibilidades es muy amplia. De ahí el factor creativo de la virtualidad. Ahora bien, lo importante es que se opte por el modelo que se opte se haga de forma institucional y se desarrolle de forma coherente.

No existe un modelo de educación virtual, de la misma forma que seria erróneo pensar que existe en la modalidad presencial. Lo que define el modelo es tan simple como determinar si se corresponde con los objetivos institucionales estratégicos que se persiguen a la vez que asegurar la coherencia con el uso de la tecnología, de la teoría o sistema de aprendizaje predominante a usar y con el marco organizativo del que vamos a dotarnos para implementarlo.

Figura 4. Modelo de análisis estratégico para la introducción de una innovación.

Esquema de análisis estratégico

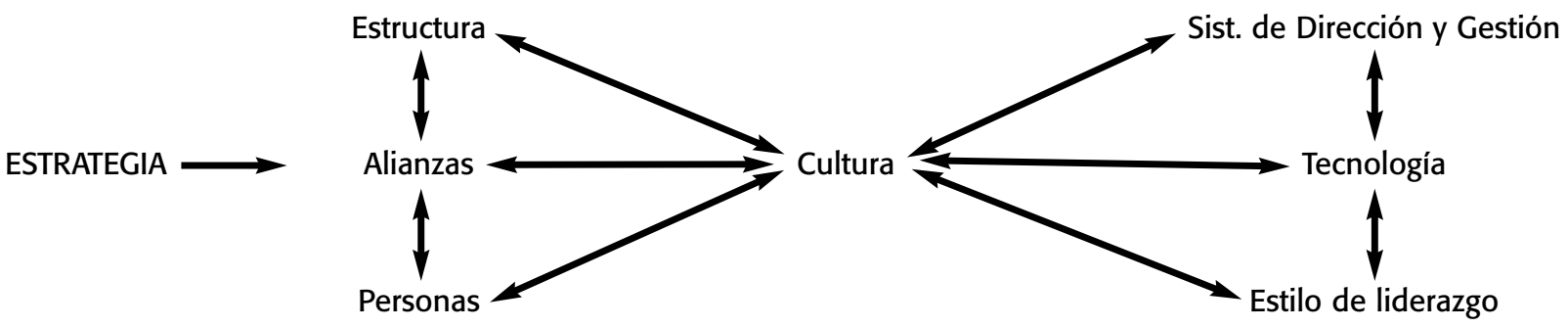




\section{FACTORES CLAVE EN LOS PROCESOS ESTRATÉGICOS DE INTRODUCCIÓN Y USO DEL E.LEARNING}

La estrategia es un arte que, al igual que otros, si bien acepta consejos estos son siempre de difícil aplicación directa. A pesar de ello, lo mejor en estos casos es aprender de lo que otros han realizado, de sus planificaciones y de sus resultados, e intentar trabajar en su adaptación a la realidad de cada institución. No existe, por tanto, una estrategia, sino diversas ${ }^{3}$. No existe tampoco unas estrategias buenas y otras malas, todo depende, como decíamos al principio, de la capacidad de entender al usuario, al estudiante, y de valorar adecuadamente sus necesidades de aprendizaje. No es una tarea fácil.

La figura 4 nos muestra algunos elementos básicos a tener en cuenta en la tarea de elaboración de una estrategia de introducción de un proceso de innovación institucional. Se trata, a su vez, de un modelo de análisis, adaptado del modelo estratégico de Stonich 4 que ampliado se compone de los ocho aspectos que se detallan a continuación:

\section{- Estrategia}

Es el resultado esperado. Distinguimos en la estrategia dos niveles, el de la formulación de la misma y su explicitación, y el de la implementación. Definimos para el modelo de análisis cada uno de los niveles de la siguiente forma

- Formular la estrategia es decidir dónde esta hoy la institución y dónde debería estar mañana. Habitualmente se explicita en los planes estratégicos institucionales

- Implementar la estrategia es decidir cómo llevar la institución desde donde hoy están hasta donde debería estar mañana. Habitualmente se concreta en los planes específicos de desarrollo.

\section{- Cultura}

Entendemos por cultura el conjunto de maneras tradicionales y habituales de pensar, sentir y reaccionar ante oportunidades y problemas con los que se enfrenta la organización; así como la pauta de creencias y expectativas, compartidas por los miembros de una organización. Sin duda uno de los elementos más complejos de analizar y a la vez más difíciles de modificar.

\section{- Estructura}

La estructura la entendemos como el particular ordenamiento de obligaciones, informaciones y de responsabilidades que se dan dentro de una institución. No es el organigrama de la institución, si no el mapa de relaciones estructurales, de responsabilidades, de procesos internos, etc. No confundir, por tanto, con la estructura formal. Ya sabemos que en las instituciones hay mucho más de lo que reflejan sus organigramas.

\section{- Personas}

La definición de los puestos de trabajo y de los perfiles profesionales es parte también de este ámbito. En la implementación de la estrategia es necesario determinar los tipos de cometidos que implican los puestos de trabajo, cualquier tipo de conocimientos especiales que requieran y qué estilo o punto de vista es el más eficaz. Como en cualquier introducción de una innovación la superación de las barreras producidas por las resistencias al cambio son determinantes. Las acciones encaminadas hacia la consecución de la transparencia y la información en todo el proceso de introducción de la innovación son determinantes.

\section{- Sistemas de dirección y gestión}

Configuran el conjunto de instrumentos de que dispone la dirección para implementar la estrategia. Constituye básicamente el sistema de dirección y de gestión de la institución. Entendemos que los principales procesos de dirección son: la planificación, la programación, el presupuesto y la valoración-retribución.

\section{- Tecnología}

La tecnología entendida como instrumento al servicio de la estrategia. La incorporación de la tecnología, adaptada a las necesidades de sus usuarios, en tanto que factor de transformación institucional. No sólo la decisión de qué tecnología debemos usar es lo que cuenta. Lo importante es el uso de la tecnología, a su vez, en la consecución de los objetivos del propio proceso de introducción de la innovación.

\section{- Alianzas}

La selección de los socios necesarios para alcanzar los objetivos institucionales forma parte del concepto de estrategia. Estos socios o aliados pueden ser tanto internos como externos a la institu- 
ción. Sin duda los aliados internos son determinantes para el éxito en la introducción de una innovación, pero también lo son las alianzas externas. Éstas pueden ayudar en muchos procesos, ya sea de valoración de la innovación, como de facilitación de la misma.

\section{- Estilo de liderazgo.}

Entendemos en este ámbito la selección de las conductas de eficacia para la dirección y la toma de decisiones los problemas adaptativos que habitualmente se presentan en las instituciones educativas. Parece obvio que la imposición no es el mejor método para la introducción de una innovación. Las instituciones educativas, en tanto que instituciones que trabajan con personas de un alto nivel intelectual y de conocimiento, necesitan procesos innovativos liderados de forma participativa y deliberativa.

Observando de nuevo la figura 4 nos damos cuenta, una vez más, de la importancia del la búsqueda del equilibrio en este tipo de procesos estratégicos. La tipología de las instituciones de educación superior así lo piden, especialmente debido al perfil de las personas que las configuran. Universidades, al igual que hospitales, son organizaciones formadas por personas con un alto nivel de capacidades intelectuales y profesionales. Obviar esto e intentar dirigir o dinamizar procesos de cambio como uno lo haría en una empresa o en una fábrica es un grave error. No estamos hablando de organizaciones empresariales, estamos hablando de organizaciones de servicio, y en su mayor parte, financiadas por fondos públicos y lideradas por personas no expertas en administración directiva. Todo un reto, sin duda.

\section{ALGUNAS LECCIONES APRENDIDAS Y TENDENCIAS A MODO DE CONCLUSIÓN}

Quizás lo más obvio que hemos observado en los estudios realizados ${ }^{5}$ es precisamente que lo habitual en los procesos de introducción de innovaciones en las instituciones de educación superior -al igual que en la mayoría de las organizaciones de servicios- es que no existe una planificación estratégica previa. Y a pesar de ello las innovaciones se introducen, pero es a costa de errores, de implicación de pocas personas, de actos voluntaristas y lo que es peor, a costa de dinero público en la mayoría de los casos. Hoy, la tendencia es a profesionalizar estos procesos y a introducir cada vez más la planificación estratégica. Esta es una gran lección aprendida que esperemos de sus frutos.

El liderazgo es determinante en cualquier proceso de cambio institucional. Si bien los cambios se estimulan des de los profesionales de cada institución, estos sedimentan institucionalmente si son asumidos y liderados por quienes tienen la responsabilidad del gobierno de la misma. La introducción y uso del e.Learning debe verse por la institución como una apuesta estratégica, y por tanto, liderada claramente por la dirección. No es la ilusión de un grupo o la moda de otro, es o debe ser una apuesta institucional. El ritmo de introducción y aceptación mejora en tanto que se actúa institucionalmente y se transmite claramente esta voluntad institucional.

Se evidencian algunas tendencias de cambio en las instituciones de educación superior que pueden ayudar a entender mejor la evolución que se esta llevando a cabo. Las agrupamos en tres grandes ámbitos: las transformaciones tecnológicas, necesarias para la introducción y uso de las tecnologías de la información y de la comunicación que dibuja la sociedad red; las transformaciones organizativas, propias de la adaptación y uso de las TIC en los procesos internos de la institución educativa; y las transformaciones pedagógicas, circunscritas al ámbito del proceso de enseñanza-aprendizaje y dando fortaleza a la instalación de modelos híbridos de interacción educativa.

\section{- Transformaciones tecnológicas}

a) de la conectividad a la portabilidad. La tendencia hoy en las instituciones de educación superior es la disminución del número de aulas de informática o de puntos fijos de conexión a la red substituida por el aumento de la cobertura inalámbrica y del uso de dispositivos portátiles para la recepción de datos (telefonía móvil o PDA por ejemplo) o con conexión a Internet (ordenadores portátiles u otros dispositivos móviles de última generación).

b) del software propietario al software libre. Las plataformas de gestión del aprendizaje (Learning Management Systems LMS) se presentan cada vez más robustas en entornos de software libre. A parte del tema del coste de las licencias de las plataformas propietarias, hecho que sin duda interviene en decisiones de cambio hacia plataformas de código abierto, otros elementos que aparece con fuerza es el de 
la cooperación interuniversitaria en el desarrollo tecnológico, por una parte, y por otra, claro está, la apuesta social de la institución universitaria por el conocimiento libre y abierto. Constatamos también un esfuerzo por la planificación estratégica en el desarrollo de los servicios tecnológicos, acompañado de la opción social que conlleva el fenómeno del código abierto. Gobiernos autonómicos en España, como el de Extremadura y Andalucía y ahora recientemente el de Cataluña ${ }^{a}$, impulsan el uso del software libre y apoyan su desarrollo en las instituciones de educación superior.

c) de las plataformas virtuales a los entornos flexibles. El conocimiento y la experiencia creciente por parte de profesores y estudiantes en el uso de las TIC por una parte, y la aparición de aplicaciones en red que apoyan el trabajo cooperativo o facilitan el acceso a la información nos muestran una evolución en el uso de la tecnología que va más allá del espacio propio de lo que convenimos en llamar "campus virtuales". Nos referimos a aplicaciones como los Blog, los Wiki, el Podcasting o el RSS, por ejemplo.

\section{- Transformaciones organizativas}

a) hacia la administración académica abierta. Se trata de garantizar el acceso a los elementos administrativos del proceso académico a cualquier hora y desde cualquier lugar. Este es un proceso que si bien afecta directamente en su implementación al personal de servicios de la organización educativa influye directamente en la vida académica de toda la institución.

b) aparición de nuevos perfiles profesionales. Resulta habitual encontrar hoy nuevos grupos de profesionales como resultado de las transformaciones ocasionadas por el uso de las tecnologías. A parte de los departamentos de informática, encontramos diseñadores instruccionales trabajando junto a los profesores, documentalistas especializados en información electrónica complementando a los profesionales habituales en las bibliotecas, desarrolladores de aplicaciones online, etc. Estos nuevos perfiles ocasionan, especialmente en las instituciones con estructuras de personal rígidas, situaciones de creatividad organizativa, así como políticas de incentivos para el desarrollo profesional de los profesionales ya en activo en la institución ${ }^{5}$. c) el emprendedurismo. Los trabajos de Clark $^{6}$ y Shattock $^{7}$ no contemplan especialmente la tecnología como elemento determinante en el desarrollo de la institución educativa emprendedora. El elemento innovador de la tecnología aplicado en instituciones flexibles y relacionadas con su entorno (stakeholders) comporta no sólo la generación de oportunidades institucionales sino también para la comunidad universitaria.

\section{- Transformaciones pedagógicas}

a) Aprendizaje flexible y la lógica de la hipertextualidad. Debemos destacar aquí los trabajos y las relevantes aportaciones de Collis y Moonen ${ }^{8}$. La tecnología facilita un proceso de aprendizaje flexible, basado en la interacción y la personalización. La creación compartida, la opción múltiple, el aprender haciendo, etc, son elementos que adquieren un papel más relevante en los procesos educativos mediados por la tecnología. La hipertextualidad muestra una nueva lógica, a la que están más habituados los estudiantes que los profesores, que permite la multidireccionalidad y la no secuencialidad. Una lógica que rompe con los esquemas unidireccionales tradicionales de la educación superior.

b) Nuevos roles docentes y discentes. No sólo se transforma el papel del profesor, que pasa a ser el de facilitador ${ }^{9}$ si no que también se modifica el papel del estudiante. Algunos estudios recientes nos muestran como en entornos flexibles de aprendizaje mediados por Internet la interacción aumenta, no sólo entre estudiante y profesor, sino que también entre la institución y los materiales interactivos multimedia. Observamos que el aprendizaje de esta nueva situación formativa puede ser más costoso para el profesor que para el estudiante, en tanto que éste forma ya parte de la sociedad red y usa sus tecnologías de forma habitual, mientras que el profesor es a menudo nuevo en estos entornos. De ahí que debamos aprovechar esta oportunidad, no sólo para formar al profesorado en el uso de las TIC -algo que ya está bastante generalizado- sino sobretodo en las nuevas metodologías educativas con uso intensivo de Internet, metodologías que corresponden a un nuevo medio comunicativo y que se fundamentan en la interacción. El resultado es evidente: profesor y estudiante se convierten a la vez en autores y actores del proceso de aprendizaje, en profesores y alumnos a la

a. En la Comunidad Autónoma de Extremadura trabajan en el proyecto LINEX http://www.campuscomputing.net/; y en la de Andalucía en el GUADALINEX http://www.guadalinex.org/modules/news 
vez, en coautores de todo material y producto formativo resultante. Ahí encontramos una de las más grandes transformaciones.

c) Open Eduactional Resources. Des de que hace años el MIT puso sus materiales en abierto han seguido su ejemplo otras instituciones universitarias. Hoy, fruto también de la cultura de código abierto con una clara implicación social propia de la institución universitaria, algunas instituciones están dando un paso más adelante y sitúan en la red no sólo los materiales de aprendizaje sino que también todos los recursos educativos de los que disponen. Esta práctica, propia de la cultura Internet, viene a facilitar las transformaciones pedagógicas en tanto que apoya el desarrollo del aprendizaje y de la docencia flexible y propicia el cambio en la organización y en el diseño educativo del proceso formativo.

Una vez más, y como la más importante lección aprendida, destacamos la necesidad de la planificación estratégica en la introducción y uso de Internet en el formación, al igual que en cualquier otro proceso formativo. El cambio de cultura y de proceder en una institución no es resultado del azar o una generación espontánea; el cambio, no nos quepa duda, se gestiona.

\section{BIBLIOGRAFÍA}

1. Holmes, B. and J. Gardner (2006). e.Learning. Concepts and Practice. London, SAGE Publications Ltd.

2. Bates, A. W. T. (2000). Managing Technological Change: Strategies for Colleges and University Leaders. San Francisco, Jossey-Bass.

3. OECD (2005). e.Learning in Tertiary Education. Where do we stand? Paris, OECD Press.

4. Stonich, P. J. (1982). Cómo implementar la estrategia. Madrid, Editorial Instituto de Empresa.

5. Duart, J. M. and F. Lupiáñez (2005) E-strategias en la introducción y uso de las TIC en la universidad. Revista de Universidad y Sociedad del Conocimiento RUSC Volume 1 Issue 4.

6. Clark, B. R. (1998). Creating Enterpreneurial Universities. Oxford, IAU Press Pergammon.

7. Shattock, M. (2003). Managing Successful Universities. Berkshire, Open University Press.

8. Collis, B. and J. Moonen (2001). Flexible Learning in a Digital World: Experiences and Expectations. London, Kogan Page.

9. Duart, J. M. and A. Sangrà (2000). Aprender en la virtualidad. Barcelona, Gedisa. 